\title{
Personalised imaging and biomechanical modelling of large vessels
}

\author{
Rob Krams · Marcel Breeuwer · Frans van de Vosse
}

Received: 12 October 2008/ Accepted: 13 October 2008/Published online: 6 November 2008

(C) The Author(s) 2008. This article is published with open access at Springerlink.com

The large blood vessels are crucial for blood flow distribution in the body but they may be subjected to disease processes like atherosclerosis, and consequences thereof like aneurysm formation, stroke, and myocardial infarction. These diseases are associated to a majority of the mortality of cardiovascular disease, which may be as high as $33 \%$ of overall disease related mortality. The function of these blood vessels has successfully been studied in the framework of biology, physics, and imaging. While a decade ago vessel function was merely described in terms of average blood flow, overall compliance and distensibility, the turbulent development of computational resources and numerical modelling has enabled calculations of local wall stress, local blood flow, and local wall deformation. Especially the methods to solve the non-linear NavierStokes equations and the equations that describe non-linear solid mechanics have evolved over the last decade and currently enable shear stress distributions in complex vessel geometries. Furthermore, the development of invasive and non-invasive imaging enables imaging the 3D geometry of blood vessels and thereby to develop personalised

\footnotetext{
R. Krams

Bioengineering, Imperial College,

Campus South Kensington, London, UK

e-mail: r.krams@imperial.ac.uk

\section{Breeuwer}

Healthcare Informatics, Philips Healthcare,

P.O. Box 10000, 5680 DA Best, The Netherlands

e-mail: marcel.breeuwer@philips.com

F. van de Vosse $(\bowtie)$

Biomedical Engineering,

Eindhoven University of Technology,

P.O. 513, $5600 \mathrm{MB}$, Eindhoven, The Netherlands

e-mail: f.n.v.d.vosse@tue.nl
}

and segmental calculations of the wall stress and shear stress fields. This Special Issue is focussed upon the development and improvement of methods to obtain personalised information of the arterial tree in terms of vascular geometry, pressure distribution, shear stress distribution and wall stress distribution, in order to predict better interventions for individual patients.

The first paper in this issue [9] gives an overview of developments in diagnostic tests for carotid and coronary disease, and aortic aneurysms and dissection. It is concluded that improved diagnostics by applying biophysics based mathematical model eventually will lead to improved patient management.

In the second series of papers the technical description of automated methods for image reconstruction is presented. In the first of the series, Peiro [8] describe methods to obtain high quality reconstructions of images of blood vessels and the consequences for mesh- generation and CFD quality. Two other papers [1, 2] describe methods to develop a cascade of methods to start from an image and end with a wall stress or shear stress field in either the carotid artery [1] and aortas [2]. These methods are essential for obtaining patient specific information in the clinical environment.

In the next series of papers, the pathophysiology of diseases is deepened by showing that haemodynamics contributes factors to the formation of brain aneurysm [3], aneurysm expansion [4] and stent-graft vessel wall interaction [5]. Chien et al. [3] show that in ruptured aneurysm the flow field is different compared to flow fields in aneurysm without rupture. The question is whether this difference could have been detected before rupture occurred. Helderman et al. [4] develop a general method to predict aneurysm expansion, while Lam et al. [5] describe the parameters that will help to better design stents. 
In the fourth series of papers, patient-specific interventions are described. Pekkan et al. [7] describe a method to obtain patient-specific information on anatomy, and flow field in patients with congenital heart defects. On top of that they simulate surgical interventions in the patientspecific environment and thereby provide useful information for surgeons. Sun et al. [10] provide similar methods for patients with complex congenital heart defects and simulate patient-specific bilateral bidirectional Glenn procedures. Due to the complexity and non-linearity of the problem these computational methods are indispensable to understand impact of intervention and help the surgeons substantially in their planning. The methods described in this issue bear direct relationship to other areas of biomedical engineering as support post-endoscopic surgery of the nasal cavity [11] and rationalizing stent design [6].

This special issue offers an overview of a rapid expanding and important field for the application of engineering principles in disease of the large vessels. The technical developments enable to study patient-specific spatial and local distributions of shear stress, wall stress and anatomy leading to a better prediction of changes induced by interventions. As such this field hold large promise to gain acceptance in diagnoses, percutaneous interventions, surgery and other medical fields.

Open Access This article is distributed under the terms of the Creative Commons Attribution Noncommercial License which permits any noncommercial use, distribution, and reproduction in any medium, provided the original author(s) and source are credited.

\section{References}

1. Antiga L, Piccinelli M, Botti L, Ene-Iordache B, Remuzzi A, Steinman DA (2008) An image-based modelling framework for high-throughput computational haemodynamics. Med Biol Eng Comput. doi: 10.1007/s11517-008-0420-1

2. Breeuwer M, de Putter S, Kose U, Speelman L, Visser K, Gerritsen F, Hoogeveen R, Krams R, van den Bosch H, Buth J,
Gunther T, Wolters B, van Dam E, van de Vosse F (2008) Towards patient-specific risk assessment of abdominal aortic aneurysm. Med Biol Eng Comput. PMID: 18810521, doi: 10.1007/s11517-008-0393-0

3. Chien A, Tateshima S, Castro M, Cebral J, Viñuela F (2008) Patient-specific flow analysis of brain aneurysms at a single location: comparison of hemodynamic characteristics in smallaneurysms. Med Biol Eng Comput. doi:10.1007/s11517008-0400-5

4. Helderman F, Manoch IJ, Breeuwer M, Kose U, Schouten O, van Sambeek MRM, Poldermans D, Pattynama PTM, Wisselink W, van der Steen AFW, Krams R (2008) A numerical model to predict abdominal aortic aneurysmexpansion based on local wall stress and stiffness Med Biol Eng Comput. doi:10.1007/ s11517-008-0358-3

5. Lam SK, Fung GSK, Cheng SWK, Chow KW (2008) A computational study on the biomechanical factors related to stentgraft models in the thoracic aorta. Med Biol Eng Comput. doi: 10.1007/s11517-008-0361-8

6. Mortier P, De Beule M, Van Loo D, Masschaele B, Verdonck P, Verhegghea B (2008) Automated generation of a finite element stent model. Med Biol Eng Comput. doi:10.1007/s11517-0080410-3

7. Pekkan K, Whited B, Kanter K, Sharma S, de Zelicourt D, Sundareswaran K, Frakes D, Rossignac J, Yoganathan AP (2008) Patient-specific surgical planning and hemodynamic computational fluid dynamics optimization through free-form haptic anatomy editing tool (SURGEM 8) Med Biol Eng Comput. doi: 10.1007/s11517-008-0377-0

8. Peiró J, Sherwin SJ, Giordana S (2008) Automatic reconstruction of a patient-specific high-order surface representation and its application to mesh generation for CFD calculations. Med Biol Eng Comput. 2008 Sep 16. PMID: 18795356, doi 10.1007/ s11517-008-0390-3 [Epub ahead of print]

9. Ricotta JJ, Pagan J, Xenos M, Alemu Y, Einav S, Bluestein D (2008) Cardiovascular disease management: the need for better diagnostics. Med Biol Eng Comput. doi:10.1007/s11517-0080416-x

10. Sun Q, Wan D, Liu J, Hong H, Liu Y, Zhu M Patient-specific computational fluid dynamic simulationof a bilateral bidirectional Glenn connection. Med Biol Eng Comput. doi:10.1007/s11517008-0376-1

11. Xiong G, Zhan J, Zuo K, Li J, Rong L, Xu G (2008) Numerical flow simulation in the post-endoscopic sinus surgery nasal cavity. Med Biol Eng Comput. doi:10.1007/s11517-008-0384-1 